\title{
Hígado: Siete Segmentos Hepáticos
}

\author{
Liver: Seven Hepatic Segments
}

César Augusto Durand López*

DURAND, L. C. A. Hígado: siete segmentos hepáticos. Int. J. Morphol., 34(4):1522-1530, 2016.

RESUMEN: Estudiamos la estructura intrahepática en 200 hígados (A05.8.01.001)* humanos: Las ramas portales, arteriales y biliares formaron siete pedículos segmentarios antes de penetrar en su parénquima correspondiente. Los cuatro primeros pertenecen, generalmente, al lóbulo izquierdo (A05.8.01.027) y los segmentos V, VI y VII al lóbulo derecho (A05.8.01.026). En un 80 \% la rama portal V es derecha y en $20 \%$ izquierda; por su parte, en un $80 \%$ la fisura portal principal (A05.8.01.035) divide el lóbulo derecho del izquierdo, extendiéndose desde la fosa de la vesícula biliar (A05.8.01.013) al margen izquierdo de la vena cava inferior (A12.3.09.001); en un 20\% la fisura portal derecha (A05.8.01.036) divide el lóbulo derecho del izquierdo, ella se extiende desde un punto equidistante entre el margen vesicular derecho y el ángulo anterior derecho del hígado, describiendo una curva de convexidad externa y alcanza el margen derecho de la vena cava inferior. En un $70 \%$ el hígado estuvo irrigado por la arteria hepática propia (A12.2.12.029), en un $30 \%$ ésta se anastomosaba con arterias hepáticas accesorias. Observamos anastomosis entre la arteria hepática derecha (A12.2.12.030) y la izquierda (A12.2.12.035) en un $55 \%$ de los casos en el hilio inferior y no en el espesor del parénquima. Las arterias segmentarias al penetrar a su correspondiente segmento se vuelven terminales. Los conductos biliares aberrantes son conductos biliares segmentarios que no han confluido para constituir el conducto hepático derecho (A05.8.01.062), drenan en el conducto hepático común (A05.8.01.061) o en el conducto cístico (A05.8.02.01 1). El tronco común biliar de los segmentos VI y VII drena en el conducto hepático izquierdo (A05.8.01.065) en el $21 \%$ de los casos, no más allá de un cm del punto de formación del conducto hepático común. En relación al grupo venoso superior, conformado por la vena hepática izquierda (A12.3.09.008), vena hepática intermedia (A12.3.09.007) y vena hepática derecha (A12.3.09.006), observamos venas del lóbulo caudado+ en el $100 \%$ de los casos y la vena hepática inferior derecha+ en el $61 \%$. Estas tenían un diámetro entre 5 y 20 mm, habiendo 5 $\%$ de casos con vena hepática media derecha+. En un $40 \%$ existe un puente parenquimal que une los segmentos III y IV. En un $25 \%$ apreciamos lóbulos hepáticos accesorios que se desprenden de la cara visceral del hígado. El objetivo de este trabajo consistió en aportar una clasificación sencilla de la segmentación hepática desde un punto de vista quirúrgico.

PALABRAS CLAVE: Segmentos hepática; Clasificación.

\section{INTRODUCCIÓN}

El hígado es un órgano altamente vascularizado, por lo cual, conocer su vasculatura es conocerlo profundamente. Para poder realizar intervenciones quirúrgicas en el hígado se debe controlar primero ciertos aspectos, tales como: ingreso de sangre al órgano y drenaje venoso. El ingreso de sangre ocurre a través del pedículo hepático inferior+ debido al aporte de la vena porta hepática (A12.3.12.001) y la arteria hepática propia. Posteriormente, el control del drenaje venoso se da a través del pedículo postero-superior.

La segmentación hepática (A05.8.01.033) ha sido propuesta anteriormente por diversos autores, sin que aún se llegue a un consenso; esta situación llevó a la International Hepato-Pancreato-Biliary Association a formalizar la división del hígado en aras de un acuerdo para la clasificación de las resecciones hepáticas, basado en la bibliografía exis- tente y no en disecciones anatómicas. En el presente estudio aportamos una clasificación sencilla desde un punto de vista quirúrgico.

\section{MATERIAL Y MÉTODO}

Estudiamos la estructura intrahepática en 200 hígados humanos procedentes de necropsias; distribuídos según se indica a continuación: Edad: Fetos $(n=4) ; 1$ mes de edad $(n=14)$; 1-6 meses $(n=33)$; 7-11 meses $(n=16) ; 1-5$ años $(n=14) ; 6-10$ años $(n=7) ; 11-20$ años $(n=30) ; 21-40$ años $(n=55) ; 41-60$ años $(n=22) ; 61-80$ años $(n=5)$. Sexo: 130 fueron varones y 70 mujeres. Raza: 165 fueron mestizos, 27 de raza blanca, 6 de raza negra y 2 de raza amarilla.

\footnotetext{
* Cirujano del Hospital de Emergencias José Casimiro Ulloa, Av. Roosevelt 6355 Miraflores, Lima, Perú. Profesor de Anatomía Humana, Facultad de Medicina, Universidad Ricardo Palma, Lima, Perú.
} 
Se llevó a cabo la inyección de acrílico en 150 hígados. En jeringas de $50 \mathrm{ml}$ se mezcló acrílico solido/líquido en partes iguales y por cada $20 \mathrm{ml}$ se agregó $1 \mathrm{~g}$ de color (azul, verde o rojo). Se inyectaron la vena porta hepática, la arteria hepática propia, el conducto biliar principal (ligando el conducto cístico) y la vena cava inferior con las venas hepáticas de drenaje. Previamente, los hígados fueron lavados durante 24 horas; después se realizó la inyección acrílica y se mantuvieron 24 horas en recipientes con agua. Después fueron sumergidos en ácido clorhídrico al $10 \%$ durante tres días, para corroer el tejido hepático y conservar los moldes de las estructuras inyectadas (Tabla I). Otros 50 hígados frescos fueron disecados de manera convencional, teniendo en cuenta los modelos inyectados previamente obtenidos.

Tabla I. Mezcla de acrílico sólido más colorante (medidas para un hígado de adulto de mayor peso).

\begin{tabular}{lc}
\hline Estructura & Cantidad $(\mathrm{ml})$ \\
\hline V. cava inferior y venas hepáticas & 120 \\
V. porta hepática & 80 \\
A. hepática & $15-20$ \\
Vías biliares & $10-15$ \\
\hline
\end{tabular}

Usamos la Terminología Anatómica Internacional. Colocamos el número identificatorio cuando citamos un elemento anatómico por primera vez en el texto, cerramos entre paréntesis los elementos inconstantes y marcamos con una + aquellos no catalogados en la Terminología Anatómica Internacional.

\section{RESULTADOS}

Los elementos vasculobiliares llegan al hilio hepático inferior (canal porta+) por diferentes rutas, pero tienen que tomar un mismo camino cuando penetran al parénquima hepático, envueltos ya sea individualmente o en conjunto, por la cápsula fibrosa perivascular (A05.8.01.055). Las ramas de la vena porta hepática, que por su caudal tienen un papel principal en la distribución segmentaria, "atraen" a las ramas arteriales y biliares y las convierten en sus satélites, formando así un determinado número de pedículos segmentarios terminales antes de penetrar en su territorio hepático correspondiente. A partir de esto, hemos identificado siete pedículos segmentarios.

Segmentación portal (A05.8.01.033): La vena porta hepática al penetrar en el hígado lo hace en relación posterior al conducto hepático común y la arteria hepática propia, con un diámetro de aproximadamente $20 \mathrm{~mm}$ en el adulto. En el canal portal+ se divide en las ramas primarias dere- cha e izquierda, que se dirigen horizontalmente a los correspondientes lóbulos del hígado. La rama derecha en el adulto tiene un diámetro de aproximadamente $16 \mathrm{~mm}$ y la izquierda de $12 \mathrm{~mm}$; la longitud de la derecha es de 1 a 3 $\mathrm{cm}$ y la de la izquierda de 3 a $4 \mathrm{~cm}$ en su porción transversa. En la extremidad izquierda del canal porta la rama izquierda hace un ángulo y se dirige hacia adelante (porción umbilical) para ocupar la fisura del ligamento redondo. La porción transversa de la rama izquierda "inicia la nomenclatura" de la segmentación hepática, con el hígado examinado por su cara visceral, y se nombra en sentido inverso al movimiento de las agujas del reloj. Las ramas secundarias portales son terminales e irrigan porciones parenquimales denominadas segmentos portales + , sin comunicación entre ellos, lo cual crea fisuras intersegmentarias, principalmente en sentido vertical. $\mathrm{La}$ rama portal izquierda suministra una rama al lóbulo caudado ( $2 \mathrm{~mm}$ de diámetro al nacer), denominándose rama segmentaria I (segmento posterior); después, en el ángulo que forman el sector transversal y el umbilical, parte una rama (4 $\mathrm{mm}$ de diámetro) destinada al área posterior de la división lateral izquierda del hígado, es la rama segmentaria II (segmento lateral del lóbulo izquierdo); la porción umbilical de la porta izquierda termina en un bulbo que se continúa con el tejido fibroso del ligamento redondo (A05.8.01.015); de la parte izquierda del bulbo se desprende una rama segmentaria (6 $\mathrm{mm}$ de diámetro) y una o dos subsegmentarias, las cuales se dirigen a la parte anterior de la división lateral izquierda, son para el segmento III (anterior lateral izquierdo). De la parte derecha del bulbo y su cara superior, salen una rama segmentaria $(6 \mathrm{~mm}$ de diámetro) y dos o tres subsegmentarias que se orientan al lóbulo cuadrado (A05.8.01.029), la mitad izquierda de la fosa de la vesícula biliar (A05.8.01.013) y para una prolongación superior, que se dirige por encima del hilio hepático inferior+ y del segmento I hacia la vena cava inferior (A12.3.09.001), correspondiendo entonces para el segmento IV (medial izquierdo).

La rama derecha de la vena porta hepática (A12.3.12.002) tiene tres ramas principales y una accesoria del segmento I. La primera es la rama segmentaria $\mathrm{V}$ (segmento medial derecho), la más voluminosa (10 mm de diámetro), que se dirige hacia arriba y atrás, rodea la vesícula biliar (A05.8.02.001) y se ubica por encima de ella para alcanzar la porción superior del hígado. La segunda rama es la rama segmentaria VI (segmento anterior lateral derecho), de menor calibre (6 $\mathrm{mm}$ de diámetro) y se dirige hacia el ángulo hepático anterior derecho y la porción derecha del hígado. Finalmente, la tercera rama corresponde a la rama segmentaria VII (segmento posterior lateral derecho), también voluminosa (8 $\mathrm{mm}$ de diámetro), y se dirige hacia la porción posterior del hígado (Fig. 1). 
Al lado de estas siete ramas segmentarias se ven unas pequeñas, que penetran en el territorio de las ramas segmentarias, son las subsegmentarias. Estas, con frecuencia, se introducen en los segmentos III y IV. En el $100 \%$ de nuestros casos hubo siete pedículos portales. Las ramas I, II, III y IV salen de la rama izquierda de la vena porta. En el $60 \%$ de los casos, el segmento I tiene, además de la rama izquierda, otra rama que proviene de la rama portal derecha, y en el $5 \%$ una rama que sale de la misma vena porta hepática antes de dividirse. Por este motivo consideramos al lóbulo caudado como un segmento mixto, por lo que si lo clasificamos como perteneciente al lóbulo izquierdo es porque siempre existió una rama de la vena porta hepática izquierda.

Las ramas portales V, VI y VII salen generalmente de la rama portal derecha, pero en el $20 \%$ de los casos la rama segmentaria $\mathrm{V}$ emerge de la porta izquierda (Fig. 2).

En el $5 \%$ de los casos la rama portal VI, sale de la rama VII y en el $4 \%$ de la V; en el $4 \%$ hubo dos ramas segmentarias VI, una que salía de la rama $\mathrm{V}$ y otra de la VII; en el $1 \%$ la rama portal VI sale de la rama V y esta de la porta izquierda; en el $1 \%$ de los casos las ramas $\mathrm{V}$ y VII salen de un tronco común y este de la porta izquierda.

Segmentación arterial: La arteria hepática propia suministra siete ramas segmentarias y cualquier arteria que irrigue el hígado puede dar una o todas las segmentarias. Observamos también que, si el hígado está irrigado por dos o más arterias diferentes, éstas se anastomosan; inclusive esto se ha apreciado entre las arterias hepáticas derecha e izquierda, en el $55 \%$ de los casos. Presumimos que esta anastomosis podría ser del $100 \%$. Advertimos que las ramas segmentarias pueden tener un origen distinto y solo su distribución en el segmento hepático correspondiente las tipifica y numera.

Destacamos que las anastomosis arteriales se observan solamente en la placa hiliar y no en el espesor del tejido hepático. Se puede decir que las arterias al penetrar a su segmento correspondiente se vuelven terminales. La arteria hepática propia en el hilio hepático inferior se divide en dos ramas desiguales: la derecha, voluminosa y larga, y la izquierda, de menor calibre y más corta que la derecha.

Generalmente, las arterias se sitúan por debajo de los conductos biliares hepáticos principales. En el $80 \%$ de los casos las ramas izquierdas se ubican debajo de la porta izquierda y en el $20 \%$, sobre ésta (situación difícil de afrontar en las hepatectomías izquier-

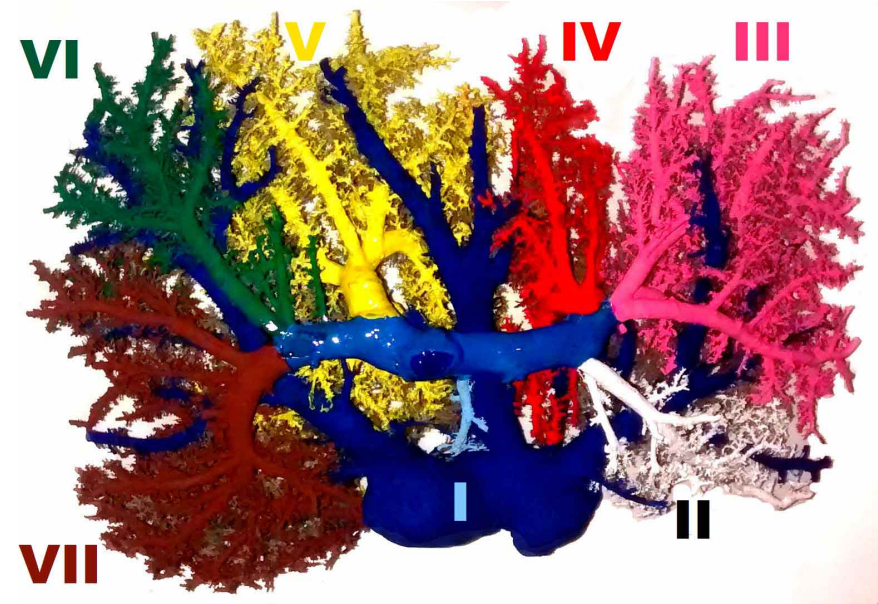

Fig. 1. La rama portal V (amarillo) es la más voluminosa originada de la porta. Ramas portales segmentarias: I en celeste, II blanco, III rosado, IV naranja, V amarillo, VI verde y VII marrón. Entre los segmentos III y IV está la vacía fisura portal izquierda, entre el IV y el V la fisura portal principal ocupada por la vena intermedia, entre el $\mathrm{V}$ y el VI-VII la fisura portal derecha con la vena hepática derecha.

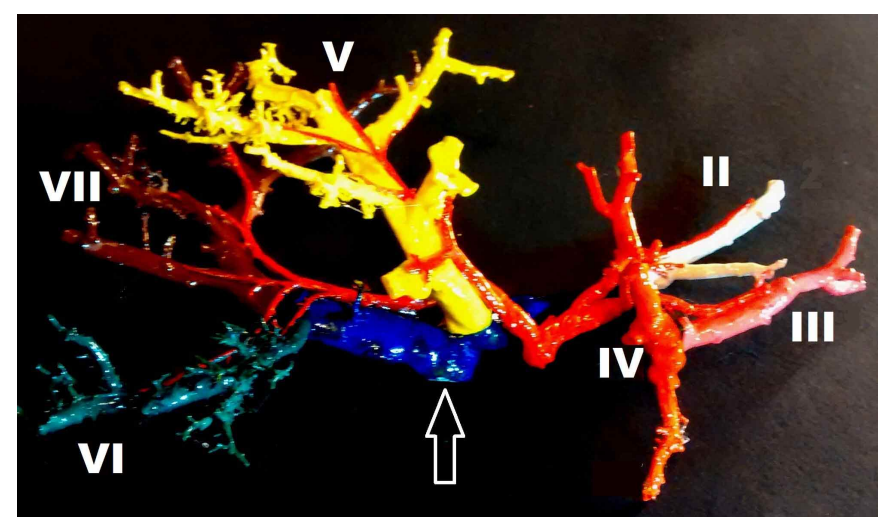

Fig. 2. Vena Porta hepática señalada con una flecha. La porta izquierda da las ramas segmentarias I, II, III, IV y V (amarillo), vista anterior.

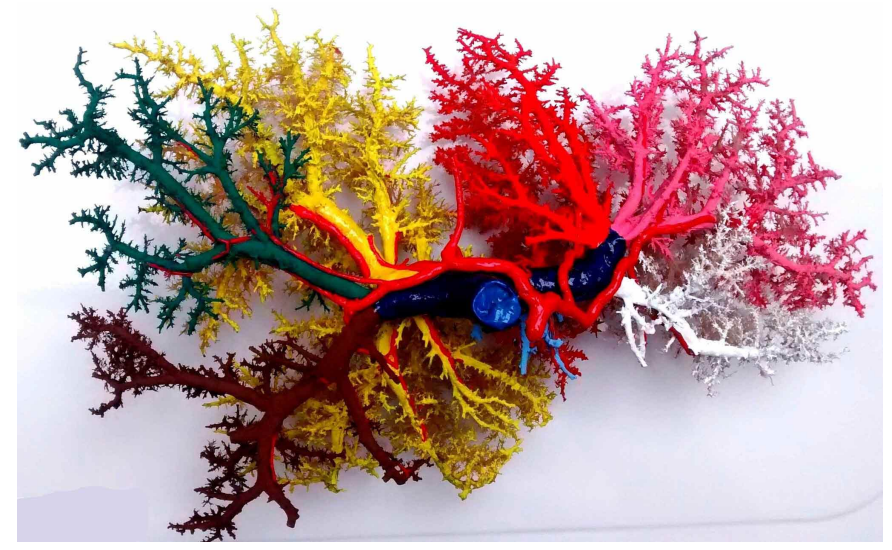

Fig. 3. En el $80 \%$ las ramas arteriales izquierdas se ubican superficialmente a la porta izquierda y en el $20 \%$, en relación profunda. Distribución común de las segmentarias de la arteria hepática propia. 
das) (Fig. 3). En la Tabla II mencionamos las distintas arterias que irrigan el hígado y en la Tabla III el origen de las ramas arteriales segmentarias. Se puede ver en la Tabla III, que algunas ramas destinadas al lóbulo izquierdo pueden salir de la arteria hepática derecha y viceversa, aunque en menor porcentaje. En la variedad más frecuente, la arteria hepática derecha irriga los segmentos I y IV.

Segmentación biliar: Generalmente, los conductos biliares I, II, III y IV constituyen el conducto hepático izquierdo y los conductos segmentarios V, VI y VII forman el derecho. Esta confluencia es más constante en el lado izquierdo, como podrá apreciarse en la Tabla IV, en la que se indica el drenaje de los troncos comunes. Se observa también que dos conductos segmentarios, ya sea en el lado derecho o izquierdo, se unen en un tronco común, el que ligado al tercer o cuarto conducto biliar del lóbulo correspondiente, formará el conducto hepático, ya sea derecho o izquierdo.

Observamos en el estudio de la segmentación biliar, que un espécimen tenía dos ramas segmentarias $\mathrm{V}$, una drenaba en el conducto hepático izquierdo y la otra en el conducto hepático común; otro especimen tenía dos ramas segmentarias VI, una se unía a la rama segmentaria V y la otra a la rama VII; un tercer especimen tenía dos conductos segmentarios VII, uno de ellos se unía al conducto $\mathrm{V}$ y el otro al VI.
Es importante advertir que las ramas segmentarias biliares derechas que desembocan en el conducto hepático izquierdo, no lo hacen más allá de un cm del punto de unión de ambos conductos hepáticos, aún en adultos (Fig. 4).

Venas de retorno hepático: La segmentación portal no coincide con la división del retorno venoso del hígado. Esto es porque en el pedículo hepático inferior+ (vena porta hepática, arteria hepática propia y conducto hepático común) no está la vena hepática eferente. Sabemos que la sangre procedente del tubo digestivo (a través de la vena porta hepática) se mezcla con la de la arteria hepática en los sinusoides hepáticos y que después de realizarse los procesos metabólicos correspondientes, esta sangre llega a la vena centrolobulillar. Las venas centrolobulillares adyacentes forman tributarias cada vez mayores, las cuales drenan en las venas hepáticas y estas se dirigen hacia el surco que en la porción posterior del hígado aloja a la vena cava inferior, en donde desembocan (hilio postero-superior).

En el sistema venoso hepático se puede distinguir un grupo venoso superior y otro inferior. El superior lo integran las venas hepáticas derechas, intermedia e izquierda. El inferior lo conforman las venas del lóbulo caudado y las venas hepáticas derechas media e inferior . Los troncos principales del sistema venoso superior se intercalan con los segmentos portales.

Tabla II. Irrigación del hígado.

\begin{tabular}{lll}
\hline \multicolumn{1}{c}{ Arterias } & $\%$ & \multicolumn{1}{c}{ Anastomosis } \\
\hline A. hepática propia & 70 & $55 \%$ entre sus ramas derecha e \\
& & $\begin{array}{l}\text { izquierda } \\
\text { Entre ambos vasos siempre } \\
\text { A. hepática propia y una rama de la a. gástrica izquierda. }\end{array}$ \\
$\begin{array}{l}\text { A. hepática propia, una rama de la a. gástrica izquierda, y una } \\
\text { rama de la a. mesentérica superior. }\end{array}$ & Mutua en todos los casos \\
A. hepática propia, y una rama de la a. mesentérica superior. & 25 & En todos los casos \\
\hline
\end{tabular}

Tabla III. Origen de las ramas segmentarias arteriales (en los casos en que el hígado estuvo irrigado solo por la a. hepática propia, 70\%).

\begin{tabular}{lccc}
\hline \multicolumn{1}{c}{ Ramas } & $\begin{array}{c}\text { A. hepática } \\
\text { izquierda (\%) }\end{array}$ & $\begin{array}{c}\text { A. hepática } \\
\text { derecha (\%) }\end{array}$ & $\begin{array}{c}\text { De ambas } \\
\text { ramas (\%) }\end{array}$ \\
\hline Segmentaria I & 30 & 16 & 24 \\
Segmentaria II & 70 & & \\
Segmentaria III & 70 & 20 & \\
Segmentaria IV & 50 & 69 & \\
Segmentaria V & 1 & 70 & \\
Segmentaria VI & & 70 & \\
Segmentaria VII & & & \\
\hline
\end{tabular}


La falta de anastomosis entre los segmentos IV y V origina la fisura portal principal. Esta comienza en la fosa de la vesicular biliar, divide el hilio a nivel de la bifurcación de la vena porta hepática y termina en el margen izquierdo de la vena cava inferior; sigue un plano de inclinación de 30 grados, abierto hacia la izquierda, con relación al plano sagital del hígado. Contiene a la vena hepática intermedia, a $4 \mathrm{~cm}$ de profundidad aproximadamente con relación al punto más alto de la convexidad hepática en el adulto.

Existen también, las fisuras hepáticas derecha e izquierda. La fisura hepática derecha empieza en un punto equidistante entre el ángulo anterior derecho del hígado y el margen vesicular derecho, después describe una curva de convexidad externa y se extiende al margen derecho de la vena cava inferior; sigue un plano de inclinación de 60 grados, abierto hacia la derecha, con relación al plano sagital del hígado. Esta fisura contiene a la vena hepática derecha, a $3 \mathrm{~cm}$ de profundidad aproximadamente con relación a la cara diafragmática del hígado en el adulto, además de las venas hepáticas derechas media e inferior cuando existen. La fisura hepática izquierda se identifica en la cara diafragmática por la inserción del ligamento falciforme (A10.1.02.303), presentando un plano de inclinación de 50 grados, abierto hacia la izquierda, con relación al plano sagital del hígado. Esta fisura no contiene ninguna vena en su porción superior; en la cara inferior del hígado se corresponde con la fisura del ligamento redondo (A05.8.01.014), la porción umbilical de la porta izquierda y la fisura del ligamento venoso (A05.8.01.010).
Las venas hepáticas derecha e izquierda desembocan simétricamente en la cara anterior y superior de la vena cava inferior y es fácil localizarlas al incidir el ligamento falciforme en su mitad posterior. Las dos hojas peritoneales que lo forman se separan por detrás, dejando entre si un espacio triangular, en cuyo ángulo derecho está ubicada la vena cava inferior con las venas hepáticas. A veces, la vena hepática derecha ocupa sólo la mitad posterior de la fisura derecha. En este caso las venas media e inferior derechas presentan gran diámetro y longitud. Las venas del grupo inferior que corresponden al lóbulo caudado y al segmento posterior derecho del hígado (segmentos I y VII), drenan directamente en la vena cava inferior. En la Tabla $\mathrm{V}$ presentamos las zonas de drenaje venoso de los segmentos portales.

En el $97 \%$ de los casos la vena hepática intermedia se unió con la vena hepática izquierda antes de desembocar en la vena cava inferior; en el $3 \%$ la vena hepática intermedia desembocó aisladamente en la cava inferior, entre las venas hepática izquierda y hepática derecha. En el $99 \%$ de los casos estuvo presente la vena hepática derecha; en el $1 \%$ estuvo ausente (en este caso, la vena hepática inferior derecha tuvo un diámetro de $20 \mathrm{~mm}$ ). En el $100 \%$ de los casos existió una vena principal del lóbulo caudado+, segmento I, de $3 \mathrm{~mm}$ de diámetro y una o dos venas accesorias de menos calibre. En el $61 \%$ hubo vena hepática inferior derecha+, con diámetros que oscilaron entre 5 y $20 \mathrm{~mm}$, habiendo además un $5 \%$ de casos con presencia de vena hepática media derecha.

Tabla IV. Drenaje de los conductos biliares segmentarios.

\begin{tabular}{lccccc}
\hline Ramas & $\begin{array}{c}\text { Conducto } \\
\text { hepático } \\
\text { derecho }(\%)\end{array}$ & $\begin{array}{c}\text { Conducto } \\
\text { hepático izquierdo } \\
(\%)\end{array}$ & $\begin{array}{c}\text { En ambos } \\
\text { conductos } \\
(\%)\end{array}$ & $\begin{array}{c}\text { Conducto } \\
\text { hepático común } \\
(\%)\end{array}$ & $\begin{array}{c}\text { Conducto } \\
\text { cístico } \\
(\%)\end{array}$ \\
\hline Segmentaria I & 7 & 70 & 23 & & \\
Tronco II-III & 76 & 24 & & & \\
Tronco III-IV & & & & 6 & 2 \\
Tronco V-VI & 5 & 21 & & 6 & \\
Tronco VI-VII & 56 & & & & \\
\hline
\end{tabular}

Tabla V. Zona de drenaje venoso de los segmentos portales.

\begin{tabular}{cccccc}
\hline Segmentos & $\begin{array}{c}\text { Vena hepática } \\
\text { Izquierda }\end{array}$ & $\begin{array}{c}\text { Vena hepática } \\
\text { Intermedia }\end{array}$ & $\begin{array}{c}\text { Vena hepática } \\
\text { derecha }\end{array}$ & $\begin{array}{c}\text { Vena hepática } \\
\text { inferior y media derechas }\end{array}$ & $\begin{array}{c}\text { Vena cava } \\
\text { inferior }\end{array}$ \\
\hline I & + & & & & \\
II & + & + & & & \\
III & + & + & + & + & \\
IV & & & + & + & + \\
V & & & + & & \\
VI & & & & \\
VII & & & & \\
(+): Zona de drenaje. & & & & \\
\hline
\end{tabular}




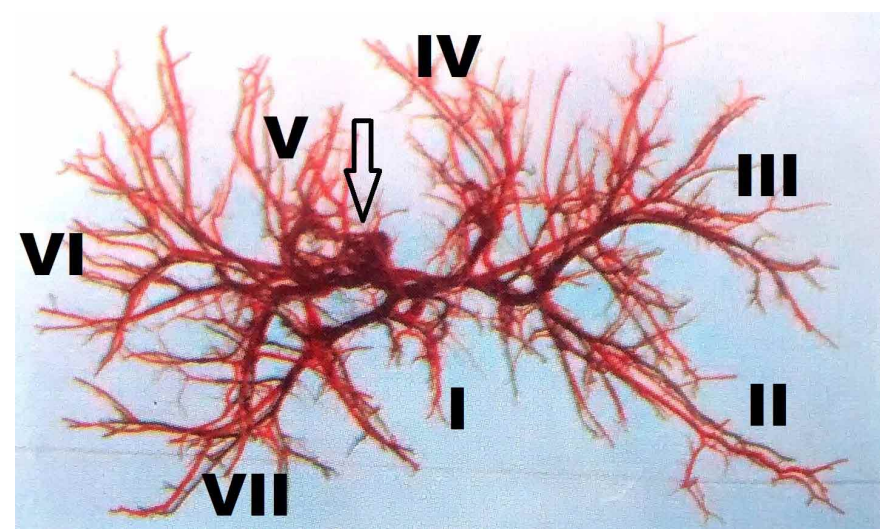

Fig. 4. Variedad frecuente de la ramificación segmentaria biliar. El Tronco común VI-VII que desemboca en el hepático izquierdo. La flecha señala el conducto hepático común.

Variaciones: En el $40 \%$ de los casos existe un puente parenquimal que une los segmentos III y IV cuya longitud fluctúa entre 10 y $80 \mathrm{~mm}$. En $25 \%$ hemos observado lóbulos hepáticos accesorios que se desprenden de la superficie hepática en la cara visceral del hígado; sus diámetros fueron desde $5 \mathrm{~mm}$ en recién nacidos, hasta $8 \mathrm{~cm}$ en adultos, siendo algunos pediculados y otros sésiles. Su importancia quirúrgica estriba en que pueden torcerse y estrangularse. Su distribución fue la siguiente: lóbulos pediculados del segmento I, $6 \%$; lóbulos sésiles del segmento I, $4 \%$; lóbulos pediculados del segmento II, $7 \%$; lóbulos sésiles del segmento II, $1 \%$; lóbulos pediculados del segmento III, $1 \%$; lóbulos pediculados del segmento IV, $5 \%$, y lóbulos pediculados del VI, $1 \%$.

\section{DISCUSIÓN}

El segmento portal+ es una porción de parénquima hepático irrigado por ramas terminales corresopndientes a la vena porta hepática y a la arteria hepática propia, que por esta inicial definición, no posee anastomosis con los segmentos vecinos, existiendo entre ellos, fisuras (principalmente verticales) que los delimitan y aíslan (unidades estructurales) y que al compartir su estrecho y constante lugar de penetración con los conductillos de drenaje biliar, forman pedículos segmentarios (denominados "portales" por ser esta vena la de mayor caudal y la de distribución previsible, que atrae a las ramas arteriales y biliares). Uno de nuestros objetivos principales fue investigar cuantos pedículos portales segmentarios nacen de las ramas portales derecha e izquierda en el hilio hepático inferior. Basamos nuestra segmentación en la distribución portal por ser de aplicación quirúrgica. Couinaud (1954) también siguió esta segmentación; pero a diferencia de él, nosotros no consideramos el segmento VIII. Couinaud describió 8 segmentos portales hepáticos. Según él, la rama portal izquierda suministra las ramas segmentarias I, II, III y IV, y la portal derecha, las ramas V, VI, VII y VIII. Nosotros no consideramos la última rama. Couinaud divide la rama portal $\mathrm{V}$, y con ello el pedículo portal (arteria y conductillo biliar), en una rama anterior que denomina "V" y otra posterior, la cual designa como "VIII". Nosotros no consideramos que exista tal distribución ya que consideramos que la rama portal $\mathrm{V}$, la más voluminosa que se origina de una rama portal primaria, después de un trayecto de tres centímetros hacia la porción superior del hígado (que es su destino) suministrando, dentro del parénquima, dos o más ramas anteriores, dos o más posteriores, dos o más laterales y dos o más mediales, siendo estas últimas las que se dirigen hacia el punto más alto de la superficie diafragmática (Fig. 1). Pero el mejor argumento que aportamos para no dividir la rama segmentaria V, en "V" y "VIII", es que esta puede nacer de la porta izquierda (Fig. 2) lo cual provocaría gran confusión si se acepta la nomenclatura hepática de 8 segmentos.

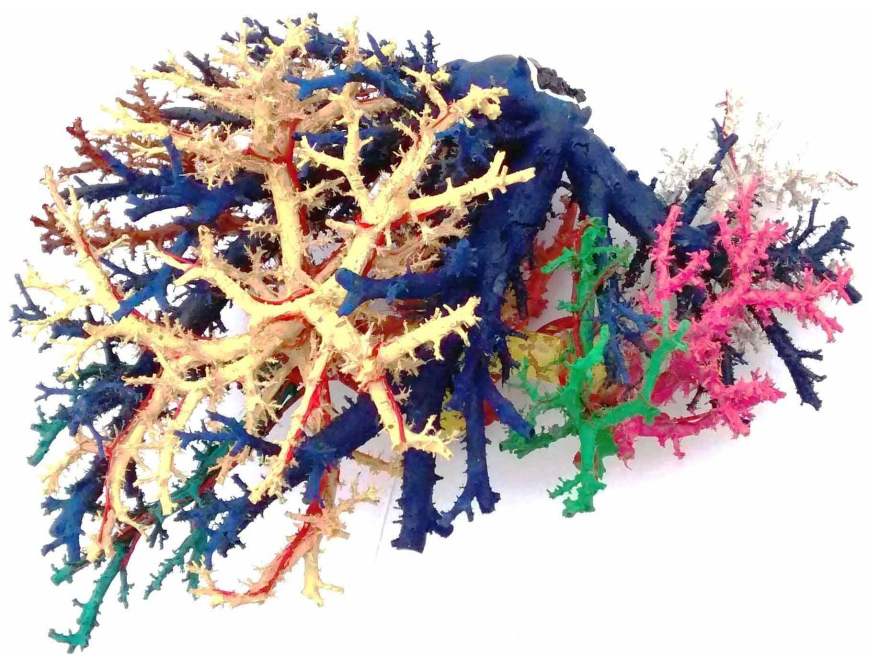

Fig. 5. La rama segmentaria $\mathrm{V}$ (amarillo) nace sola y se dirige hacia la porción superior del hígado, dando intraparenquimalmente, en forma alternada, ramas anteriores, posteriores, laterales y mediales.

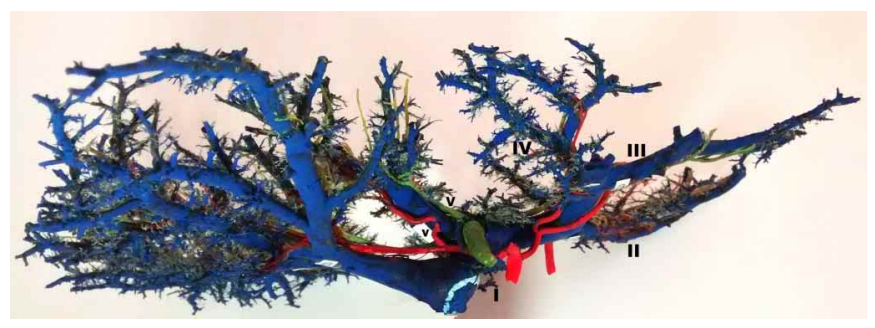

Fig. 6. Rama segmentaria $\mathrm{V}$ que nace de la porta izquierda. Cara inferior del hígado. 
Healey \& Schroy (1953) en 150 moldes hepáticos por corrosión, distinguieron cuatro segmentos principales que son divididos en un área superior y otra inferior, las que unidas al lóbulo caudado totalizaban 9 segmentos. Hidayet et al. (1977), de sus estudios en fetos y adultos, llegó a la conclusión de que existen nueve segmentos hepáticos.

En nuestras muestras no vemos posibilidad de que los segmentos principales de Healey et al. (1953) o de Hidayet et al. puedan dividirse en un área superior y otra inferior, ya que no existe dicotomización de las ramas portales, y con ello del pedículo en sí. A partir de nuestro resultados, consideramos que las ramas primarias portales tiene un mayor desarrollo que las secundarias, las cuales van saliendo alternadamente, siendo su ramificación de tipo monopódico. Al considerar como ejemplo la rama anterior derecha, de la clasificación de Healey et al., podemos ver que tiene un tallo principal, el cual se dirige hacia la porción superior del hígado, proporcionando en forma alternada ramas anteriores, posteriores, laterales y mediales (Fig. 5). Así esta rama y con ella este segmento, no puede ser subdividido en una rama superior y otra inferior. Este mismo tronco anterior de Healey et al. es dividido por Couinaud en segmentos "V" y "VIII", y por consiguiente con el mismo argumento lo rebatimos. Y si así lo fuera, lo que cuenta para nosotros no es su postrera división intraparenquimal, sino las ramas que emergen directamente de la vena porta hepática, hemos observado solamente la rama $\mathrm{V}$.

Pero lo que no han manifestado ambos investigadores, es que este tronco anterior de Healey o segmentos "V" y "VIII" de Couinaud, puede nacer de la porta izquierda (Fig. 6), con lo cual su nomenclatura se derrumba, y lo que es peor, confunde al cirujano y al radiólogo. Si se acepta nuestra nomenclatura de siete segmentos, se puede decir lo siguiente: "La rama portal $\mathrm{V}$, en el $80 \%$ de los casos emerge de la porta derecha y en el $20 \%$ de casos de la porta izquierda". Esto establece otro concepto, en caso de que la rama $\mathrm{V}$ nazca de la porta derecha, la división entre lóbulo derecho e izquierdo será una línea que se dirija desde la fosa de la vesícula biliar, al margen izquierdo de la vena cava inferior; si nace de la porta izquierda, la línea que divide ambos lóbulos será aquella que empieza en un punto equidistante entre el margen vesicular derecho y el ángulo anterior derecho del hígado, describiendo una curva de convexidad externa y alcanzando el margen derecho de la vena cava inferior. En este caso, el lóbulo izquierdo del hígado es mayor que el derecho. En nuestros moldes por corrosión de hígados de fetos y recién nacidos, observamos que las ramas portales existen ya desde estas etapas tempranas del desarrollo.

Michels (1955) describió que la irrigación del hígado por la arteria hepática propia se da solamente en el $55 \%$ de los casos. En nuestra investigación, el hígado estuvo irrigado por la arteria hepática propia en el $70 \%$ de las muestras. Conforme a la Tabla II se puede ver que, ya sea una rama de la arteria gástrica izquierda (A12.2.12.013) o una de la arteria mesentérica superior (A12.2.12.053), éstas contribuyen de manera accesoria en la irrigación del hígado, existiendo anastomosis entre ellas. Calne (1969) y Michels describieron la presencia de una rama dependiente de la arteria mesentérica superior en el 15 y $17 \%$ de sus casos, respectivamente. Michels refirió, en su estudio sobre 200 disecciones, que existen al menos 26 rutas de irrigación colateral al hígado, éstas comprenden: 10 vías por arterias hepáticas aberrantes, 6 por cortocircuitos a través del omento mayor (A10.1.02.201), el omento menor (A10.1.02.101) y vías pancreáticas, y 10 por arterias que no salen del tronco celíaco (A12.2.12.012). Las anastomosis arteriales únicamente tienen lugar en el hilio hepático inferior, mientras que las arterias segmentarias se comportan como terminales al penetrar a su segmento correspondiente. En los moldes por corrosión presentados en este trabajo no se observan anastomosis entre las arterias segmentarias. Entre los los segmentos IV y V, y I y VII existen abundantes anastomosis hiliares, pudiendo significar esto la justificación del mayor éxito resultante de la ligadura unilateral de la arteria hepática derecha o izquierda entre estos segmentos.

Generalmente los segmentos hepáticos tienen una sola rama segmentaria arterial, pero hay casos en que presentan dos arterias segmentarias, ya provengan de la arteria hepática propia o de las arterias accesorias.

Luschka (1863) describió los conductos biliares aberrantes y dijo: "Estos conductos son más o menos similares en tamaño y estructura a los conductos hepáticos". En 1927, Halpert describió tales conductos como estructuras de tipo ductal encontradas ocasionalmente en la pared vesicular, presentándose con más frecuencia en la cara visceral, de manera usual a lo largo de los márgenes de la fosa de la vesícula biliar, con una pared propia, similar a la estructura histológica de los conductos biliares intrahepáticos, con los cuales establecían comunicación. Aird (1957), sostuvo que un conducto hepático derecho accesorio de tamaño demostrable, se halló presente en el $14 \%$ de los sujetos; Missen (1969) en su trabajo los denominó conductos hepáticos accesorios.

Según nuestras investigaciones, estos conductos no son biliares accesorios, sino biliares segmentarios que no han confluido para constituir el conducto hepático derecho, los cuales vierten su secreción en el conducto hepático común o en el conducto cístico. En la Tabla IV, se puede observar que el tronco común biliar de los segmentos V y VI desemboca en el conducto hepático común en el $1 \%$ de los casos; el tronco común VI y VII desemboca en el conducto hepático 
común en el $6 \%$ de los casos y en el conducto cístico en el 2 $\%$; en estos el "hepático derecho" está constituido, generalmente, por el conducto segmentario más voluminoso, el V. Pero, paradójicamente, no es tan voluminoso como pudiera creerse, solo tiene un diámetro de 2 a $3 \mathrm{~mm}$.

Podemos decir que no hay anastomosis entre las tributarias derecha e izquierda de los conductos hepáticos (Tolino et al., 2010). Sólo a través del conducto segmentario I, que no aboca en el conducto hepático derecho, sino en el tronco común de los segmentos VI y VII, se observa esta anastomosis. Pero ya sabemos, según la Tabla IV, con qué frecuencia el tronco VI y VII desemboca en el conducto hepático izquierdo. Es decir, el segmento drena el lado derecho, pero vuelve al conducto hepático izquierdo.

En el $21 \%$ de los especímenes de Healey \& Schroy, el segmento posterior biliar drena en el conducto hepático izquierdo y en el $7 \%$ el segmento anterior. En nuestro trabajo, el tronco común biliar de los segmentos VI y VII drena en el conducto hepático izquierdo en el $21 \%$ de casos.
En cuanto al drenaje venoso, notamos que el segmento VI drena, generalmente, en la vena hepática derecha, pero si esta no tiene una longitud suficiente, la vena hepática intermedia tiende a alargarse hacia dicho segmento. De este hecho resulta que el segmento VI (derecho) pueda drenar a través de la vena hepática intermedia en la vena hepática izquierda; afortunadamente, el segmento VI, como puede observarse en la Tabla $\mathrm{V}$, también drena en las venas hepáticas inferiores derechas.

Ashby (1969) observó dos casos de lóbulos hepáticos accesorios; nosotros los apreciamos en el $25 \%$ de nuestros hígados, ubicados en la cara visceral; su importancia radica en que pueden torcerse y estrangularse.

Finalmente, se observó que no hubo diferencia en la segmentación hepática en cuanto a edad y sexo. El hígado tiene siete segmentos portales, cada uno corresponde a una o más ramas del sistema portal, biliar y arterial y tiene un drenaje venoso en una o dos de las tres principales venas que discurren por las fisuras portales.

DURAND, L. C. A. Liver: seven hepatic segments. Int. J. Morphol., 34(4):1522-1530, 2016.

SUMMARY: Intrahepatic structure study in 200 human livers: The Portal, arterial and biliary branches form seven segmental pedicles before entering the corresponding parenchyma. The first four belong, generally to the left lobe and the number V, VI and VII to the right. In $80 \%$ portal branch V is right and $20 \%$ is left, so in $80 \%$ the line dividing both hemi livers ranges from biliary cystic fossa to the left edge of the inferior cava vein; in $20 \%$ said line running from a equidistant point between biliary cystic fossa right margin and the right anterior angle edge of the liver, describes a convex outer curve and reaches the right edge of the inferior cava vein. $70 \%$ of the livers were supplied by the hepatic artery, in $30 \%$ there were anastomosis with accessory hepatic arteries. We observed anastomosis between the left and right hepatic artery in $55 \%$ of cases in the hilum plate and not in the thickness of the parenchyma. Segmental arteries while penetrating their segments, become terminal. Aberrant bile ducts are segmental bile, they have not come together to form the right hepatic duct, draining into the common hepatic or cystic. Biliary common duct segments VI and VII drains into the left hepatic duct in 21 $\%$ of cases, no more than $1 \mathrm{~cm}$ from the point of formation of the common hepatic. Besides in (left hepatic, intermediate and and right hepatic) upper venous system we observed veins in the caudate lobe in $100 \%$ of cases, and right lower hepatic in $61 \%$ of cases, these had a diameter between 5 and $20 \mathrm{~mm}$, and $5 \%$ with right middle hepatic. At $40 \%$ there is a parenchymal bridge linking segments III and IV. In $25 \%$ we appreciate accessory lobes arising from the underside of the liver. The aim of this study was to provide a simple classification of the hepatic segmentation from a surgical point of view.

KEY WORDS: Hepatic segments; Classification.

\section{REFERENCIAS BIBLIOGRÁFICAS}

Aird, I. A Companion in Surgical Studies. Edinburgh, Livingstone, 1957. p. 960.

Ashby, E. C. Accessory liver lobe attached to the gall-bladder. $\mathrm{Br}$. J. Surg., 56(4):311-2, 1969.

Belgihiti, J.; Clavien, P. A. \& Gadzijev. The Brisbane 2000 terminology of liver anatomy and resections. H. P. B., 2(3):3339, 2000.
Calne, R. Y. Surgical aspects of clinical liver transplantation in 14 cases. Br. J. Surg., 56(10):729-36, 1969.

Couinaud, C. Liver lobes and segments: notes on the anatomical architecture and surgery of the liver. Presse Med., 62(33):70912, 1954.

Halpert, B. Morphological studies on the gall bladder. Bull. Johns Hopk. Hosp., 41:77, 1927. 
Healey, J. E. Jr. \& Schroy, P. C. Anatomy of the biliary ducts within the human liver; analysis of the prevailing pattern of branchings and the major variations of the biliary ducts. A. M. A. Arch. Surg., 66(5):599-616, 1953.

Healey, J. E. Jr.; Schroy, P. C. \& Sorensen, R. J. The intrahepatic distribution of the hepatic artery in man. J. Int. Coll. Surg., 20(2):133-48, 1953.

Hidayet, M. A.; Wahid, H. A.; Hidayet, A. M. \& Hidayet, N. M. A study of the intrahepatic vasculature in the human fetus, in the normal adult and in adults with portal cirrhosis. Surg. Gynecol. Obstet., 145(3):378-88, 1977.

Luschka, H. Die Anatomie des Menschen. Tiibingen, 1863.

Michels, N. A. Blood Supply and Anatomy of the Upper Abdominal Organs. Philadelphia, Lippincott, 1955.

Missen, A. J. Aberrations of the biliary passages on the surface of the liver and gall-bladder and in the gall-bladder wall. $B r . J$. Surg., 56(6):427-31, 1969.

Tolino, M. J.; Tartaglione, A. S.; Sturletti, C. D. \& García, M. I. Anatomy variations of biliary tree. Surgical Implicance. Int. J. Morphol., 28(4):1235-40, 2010.
Dirección de Correspondecia

César Augusto Durand López*

Cirujano del Hospital de Emergencias "José Casimiro Ulloa"

Profesor de la Cátedra de Anatomía Humana

Facultad de Medicina

Universidad Ricardo Palma

Hospital Emergencias

Av. Roosevelt 6355

Miraflores,

Lima

PERÚ

Email: cesaraugustoo1@gmail.com

Recibido : 27-01-2016

Aceptado: 16-09-2016 